Pesq. Vet. Bras. 30(3):229-236, março 2010

\title{
Resistência anti-helmíntica de nematóides gastrintestinais em ovinos, Mato Grosso do Sul ${ }^{1}$
}

\author{
Eurico A. Sczesny-Moraes²*, Ivo Bianchin ${ }^{3}$, Karina F. da Silva ${ }^{4}$, João Batista \\ Catto $^{3}$, Michael Robin Honer ${ }^{5}$ e Fernando Paiva ${ }^{6}$
}

\begin{abstract}
Sczesny-Moraes E.A., Bianchin I., Silva K.F., Catto J.B., Honer M.R. \& Paiva F. 2010. [Anthelmintic resistance of gastrointestinal nematodes in sheep, Mato Grosso do Sul, Brazil.] Resistência anti-helmíntica de nematóides gastrintestinais em ovinos, Mato Grosso do Sul. Pesquisa Veterinária Brasileira 30(3):229-236. Departamento de Patologia, Centro de Ciências Biológicas e da Saúde, Universidade Federal de Mato Grosso do Sul, Cidade Universitária, Campo Grande, MS 79070-900, Brazil. E-mail: eurico_sczesny @ hotmail.com

Among the methods of control of gastrointestinal worms in sheep, the use of chemicals is the most common. However, the continued, and indiscriminate, use of these products has selected populations of resistant helminths to anthelmintics, a phenomenon reported in the whole world. This study aimed to identify the species of gastrointestinal parasites and diagnose the status of anthelmintic resistance in sheep in the State of Mato Grosso do Sul Brazil. Feacal egg count reduction tests (FECRT) were performed in flocks of sixteen farms, and the seven formulations used contained the following pharmacological bases: Albendazole, Ivermectin, Levamizol, Trichlorfon, Moxidectin, Closantel and one containing the first three in association. The species identified at necropsy, in adult sheep, were: Haemonchus contortus, Trichostrongylus colubriformis, Cooperia curticei, C. punctata, C. pectinata and Oesophagostomum columbianum, in order of prevalence. The formulations containing Albendazole and Ivermectin did not show efficacy in reducing the EPG in the flocks tested, with average reductions of 0.7 and $-19.6 \%$, respectively. Closantel presented an average efficacy of 6.7\%; Levamisolee, Moxidectin and Trichlorfon, 28.7, 26.8 and 65\% respectively, the combination of three bases (Albendazole, Ivermectin and Levamizol), an average efficacy of $55.8 \%$. The average percentages of infective larvae recovered in the faecal cultures, pre and post treatment were similar, indicating that resistance to the bases tested is present in all species cited, to a greater or lesser degree. The two genera predominantly resistant are Haemonchus sp., with $86.9 \%$, followed by Trichostrongylus sp., with an average of $47.5 \%$, Strongyloides sp. 33.6\%, Oesophagostomum sp. $21.4 \%$ and Cooperia sp. $19.7 \%$.
\end{abstract}

INDEX TERMS: Parasites, Haemonchus, treatments, control, small ruminants.

\footnotetext{
${ }^{1}$ Recebido em 30 de setembro de 2009.

Aceito para publicação em 6 de outubro de 2009.

2 Pós-Graduando em Ciência Animal, Departamento de Patologia, Centro de Ciências Biológicas e da Saúde, Universidade Federal de Mato Grosso do Sul (UFMS), Cidade Universitária s/n, Cx. Postal 549, Campo Grande, MS 79070-900, Brasil. *Autor para correspondência: eurico_sczesny@ hotmail.com

${ }^{3}$ Embrapa Gado de Corte, BR 262 Km 4, Cx. Postal 154, Campo Grande, MS 79002-970.

${ }^{4}$ Embrapa Gado de Corte, Campo Grande, MS. Bolsista do CNPq.

${ }_{5}^{5}$ Programa de Biotecnologia, Universidade Católica Dom Bosco, Rua 55, no 145, Vila Nova, Campo Grande, MS 79104-780.

6 Departamento de Patologia, Centro de Ciências Biológicas e da Saúde, UFMS, Campo Grande, MS.
}

RESUMO.- Entre os métodos de controle da verminose gastrintestinal em ovinos, a utilização de produtos químicos é o mais empregado. Porém, o uso indiscriminado e continuado desses produtos tem selecionado populações de helmintos resistentes aos anti-helmínticos, fenômeno relatado no mundo todo. Este trabalho teve como objetivo identificar as espécies de parasitos gastrintestinais e diagnosticar a situação da resistência anti-helmíntica em ovinos no Estado de Mato Grosso do Sul. Foram realizados testes de redução na contagem de ovos por grama de fezes (OPG) em rebanhos de dezesseis propriedades rurais; as sete formulações utilizadas continham as seguintes bases farmacológicas: Alben- 
dazol, Ivermectina, Levamisole, Triclorfon, Moxidectina, Closantel e uma contendo as três primeiras associadas. As espécies identificadas nas necropsias, em ovinos adultos, foram: Haemonchus contortus, Trichostrongylus colubriformis, Cooperia curticei, C. punctata, C. pectinata e Oesophagostomum columbianum; em ordem de prevalência. As formulações contendo Albendazol e Ivermectina não apresentaram eficácia na redução de OPG nos rebanhos testados, com médias de redução de 0,7 e $-19,6 \%$, respectivamente. Closantel apresentou eficácia média de 6,7\%; Levamisole, Moxidectina e Triclorfon de 28,7, 26,8 e 65\%, respectivamente; a associação das três bases (Albendazol, Ivermectina e Levamisole), uma média de eficácia de $55,8 \%$. As percentagens médias de larvas infectantes recuperadas nas coproculturas, tanto no pré como no pós-tratamento, foram semelhantes; indicando que a resistência às bases testadas está presente em todas as espécies citadas, em maior ou menor intensidade. Os dois gêneros predominantemente resistentes são Haemonchus sp., com 86,9\%; seguido por Trichostrongylus sp., com média de 47,5\%; Strongyloides sp. 33,6\%; Oesophagostomum, sp. $21,4 \%$ e Cooperia sp. $19,7 \%$.

TERMOS DE INDEXAÇÃO: Parasitas, Haemonchus, tratamentos, controle, pequenos ruminantes.

\section{INTRODUÇÃO}

O Brasil possui um rebanho com mais de 16,2 milhões de ovinos; as áreas com maior concentração são: Nordeste (9.286.258 cabeças), Sul (4.603.241 cabeças) e CentroOeste (1.086.238 cabeças). Mato Grosso do Sul possui o maior rebanho da região Centro-Oeste, com 464.851 cabeças, tendo apresentado um aumento de 5,7\% do rebanho, no período de 2005 a 2007 (IBGE 2007).

No aspecto sanitário a verminose gastrintestinal é o principal problema enfrentado pela ovinocultura. A verminose se apresenta sob a forma aguda, levando os animais rapidamente à morte; ou sob a forma crônica, em que os efeitos do parasitismo são gradativamente notados, tais como menor desenvolvimento corporal, perda de peso, redução na produção e na qualidade de lã, má eficiência reprodutiva, reduzida resistência a enfermidades e elevado índice de mortalidade, principalmente entre os animais jovens (Jardim 1974, Hammerberg 1986, Holmes 1987, Gennari et al. 1991). Echevarria (1988) relatou que a verminose em ovinos pode reduzir de 20 a $60 \%$ o ganho de peso e ocasionar uma taxa de mortalidade que pode variar de 20 a $40 \%$ dos rebanhos.

O controle das helmintoses com a utilização de produtos químicos é o método mais empregado. Entretanto, o uso indiscriminado e repetitivo de esquemas de tratamento tem como consequência a seleção de populações de helmintos resistentes aos diferentes grupos químicos (Amarante et al. 1992).

Esse fenômeno vem sendo relatado por diversos autores no mundo todo, inclusive no Brasil, espalhando-se lentamente por todos os Estados que apresentam cria- ções representativas de ovinos. Os primeiros registros de resistência a anti-helmínticos surgiram nos anos 60 , sendo relatado no Brasil, em primeiro lugar no Rio Grande do Sul por Santos \& Gonçalves (1967/68), que constataram a resistência de Haemonchus contortus ao Thiabendazole. Após esse relato, vários autores descreveram uma generalização do problema da resistência em criações de ovinos nas diversas regiões do Brasil: Sul (Santiago \& Costa 1979, Soccol et al. 1996, Cunha-Filho et al. 1998, Ramos et al. 2002), Sudeste (Verissímo et al. 2002), Nordeste (Vieira et al. 1989, Melo et al. 1998, Barreto \& Silva 1999, Barreto et al. 2002, Bispo et al. 2002), entre outros.

Em revisão publicada pela FAO (2003), a resistência a anti-helmínticos em ovinos foi considerada como amplamente disseminada, constituindo-se em um sério obstáculo para o controle efetivo das infecções por helmintos. Apesar do aumento significativo da ovinocultura no estado do Mato Grosso do Sul, não há estudos sobre as espécies de helmintos gastrintestinais que ocorrem na região e tampouco sobre a ocorrência de resistência aos anti-helmínticos.

Considerando estes aspectos, este trabalho teve como objetivo identificar as espécies de parasitos gastrintestinais de ovinos e diagnosticar a situação da resistência antihelmíntica em 12 municípios do estado de Mato Grosso do Sul.

\section{MATERIAL E MÉTODOS}

\section{Necropsia e identificação de espécies}

As necropsias foram realizadas entre agosto de 2006 e abril de 2007 em 25 animais adultos jovens oriundos de várias regiões do Estado e abatidos em um frigorífico comercial, na cidade de Campo Grande, MS. O abomaso, intestino delgado e grosso foram amarrados nas extremidades, retirados, abertos e lavados. No laboratório o conteúdo total foi examinado sob microscópio estereoscópico, e os helmintos presentes fixados em solução de formol a $5 \%$ e mantidos em álcool $70^{\circ} \mathrm{GL}$. A identificação das espécies foi realizada, após diafanização em fenol alcoólico, considerando os caracteres propostos por Lichtenfels \& Pritchard (1982).

\section{Regiões de estudo, formação dos lotes e tratamentos}

Dezesseis propriedades localizadas em 12 municípios de diferentes regiões do Estado foram visitadas. As propriedades estão localizadas nos seguintes municípios: Angélica, Camapuã, Campo Grande, Corumbá, Coxim, Ivinhema, Miranda, Porto Murtinho, Ribas do Rio Pardo, São Gabriel do Oeste, Sidrolândia e Terenos. Nos municípios de Camapuã, Campo Grande, Miranda e Porto Murtinho foram visitadas duas propriedades, nos demais apenas uma.

Para a escolha das propriedades, além da localização geográfica, foram considerados: a presença de rebanho ovino em número necessário para os testes, facilidades de manejo e os animais não terem recebido tratamento com anti-helmínticos há, pelo menos, quarenta e cinco dias. Para formação dos oito lotes experimentais, em cada rebanho eram coletadas amostras de fezes em aproximadamente 120 ovinos, de diferentes categorias de idade e devidamente identificados. Frente aos resultados da contagem de ovos por grama de fezes (OPG), os lotes eram formados com no mínimo dez e no máximo quinze 
animais cada, usando o método Greek Guard, para assegurar homogeneidade entre os lotes.

Em seguida os animais eram pesados para determinação da respectiva dose, aplicada na quantidade recomendada pelo fabricante de cada produto $(\mathrm{mL} / \mathrm{Kg})$ : Albendazol ${ }^{7}, 1 / 20$; Albendazol + Ivermectina + Levamisole $^{8}, 1 / 4$ Closantel $^{9}, 1 / 10$; Ivermectina $^{10}, 1 / 4$; Levamisole ${ }^{11}, 1 / 10 ;$ Moxidectina $^{12}, 1 / 10$; Triclorfon $^{13}, 1 / 1$.

A segunda visita era realizada, entre 7 a 10 dias após a dosificação. Além da contagem de OPG, foram realizadas coproculturas com o objetivo de identificar os gêneros de helmintos presentes antes e depois dos tratamentos.

\section{Diagnóstico parasitológico}

As amostras de fezes foram coletadas diretamente do reto dos animais e a contagem de OPG determinada segundo a técnica de Gordon \& Whitlock (1939) modificada, onde cada ovo contado correspondeu a 100 ovos por grama de fezes.

Para obtenção de larvas infectantes, dos diferentes gêneros parasitas, utilizou-se a técnica de coprocultura descrita por Roberts \& O'Sullivan (1950) modificada: as amostras de fezes do mesmo lote eram homogeneizadas e adicionadas com igual volume de vermiculita com granulação fina, mantidas durante sete dias em estufa do tipo B.O.D. à temperatura de $27^{\circ} \mathrm{C}$ e umidade relativa de aproximadamente $70 \%$. As larvas recuperadas foram identificadas ao nível de gênero, utilizando as chaves de identificação propostas por Keith (1953).

Teste de redução na contagem de ovos pós-tratamento e critérios de resistência e sensibilidade

A eficácia anti-helmíntica das formulações foi estimada de acordo com o programa RESO (Analysis Program, Version 2.01, CSIRO, Division of Animal Health, Glebe, NSW, Australia), onde é utilizada a média aritmética de OPG para o grupo controle e grupo tratado, calculando o intervalo de confiança das médias. Considerou-se a existência de resistência anti-helmíntica quando a redução de OPG, observada no pós-tratamento, era inferior $95 \%$ e o intervalo de confiança menor que $90 \%$; conforme recomendado por Presidente (1985), Wood et al. (1995) e Coles et al. (1992). Com os resultados das coproculturas calculou-se a condição de sensibilidade/resistência dos diferentes gêneros de helmintos presentes na área de estudo.

\section{RESULTADOS}

\section{Espécies de helmintos gastrintestinais de ovinos na região do estudo}

Foram identificadas seis espécies de helmintos nas necropsias realizadas em animais adultos: Cooperia curticei, Cooperia pectinata, Cooperia punctata, Haemonchus contortus, Oesophagostomum columbianum e Trichostrongylus colubriformis. A espécie de maior prevalência foi $H$. contortus, com $73,4 \%$. Em seguida, $T$. colubriformis $(9,9 \%)$, C. curticei $(7,2 \%)$, C. punctata $(5,0 \%)$, C. pectinata $(4,4 \%)$ e $O$. columbianum $(0,2 \%)$.

\section{Gêneros de helmintos gastrintestinais identificados nas coproculturas}

Em relação às duas espécies de maior prevalência, os resultados encontrados nas necropsias foram semelhan- tes ao encontrado na identificação de larvas nas coproculturas, onde $H$. contortus apresentou média de $80,3 \%$ e Trichostrongylus sp. 9,3\% (Quadro 1). Apenas nos rebanhos identificados como Angélica, Ivinhema, Ribas do Rio Pardo e Campo Grande (Faz.2) as percentagens de larvas de Haemonchus sp., foram inferiores à media.

Quadro 1. Contagem média de ovos do tipo Strongylida por grama de fezes (OPG) nos rebanhos testados e a percentagem dos gêneros de nematóides identificados nas coproculturas, antes dos tratamentos; HAE: Haemonchus sp., TRI: Trichostrongylus sp., STR: Strongyloides sp., OES: Oesophagostomum sp., COO: Cooperia sp.

\begin{tabular}{lcccccc}
\hline Rebanhos & OPG & HAE & TRI & STR & OES & COO \\
\hline Angélica & 996 & 35,3 & 0,8 & 49,8 & 0 & 14,3 \\
Camapuã (Faz.1) & 969 & 82,1 & 15,3 & 0 & 1,9 & 0,8 \\
Camapuã (Faz.2) & 651 & 98,4 & 1,1 & 0 & 0 & 0,5 \\
Campo Grande (Faz.1) & 1.781 & 86,5 & 13,0 & 0 & 0,5 & 0 \\
Campo Grande (Faz.2) & 1.110 & 50,2 & 27,7 & 9,0 & 7,2 & 5,9 \\
Corumbá & 3.968 & 93,0 & 0,3 & 0 & 6,7 & 0 \\
Coxim & 2.308 & 95,3 & 4,8 & 0 & 0 & 0 \\
Ivinhema & 2.731 & 67,4 & 9,3 & 10,3 & 7,0 & 6,1 \\
Miranda (Faz.1) & 877 & 81,3 & 5,0 & 1,1 & 4,0 & 8,6 \\
Miranda (Faz.2) & 692 & 88,8 & 7,9 & 0,6 & 0,8 & 2,0 \\
Porto Murtinho (Faz.1) & 5.893 & 100 & 0 & 0 & 0 & 0 \\
Porto Murtinho (Faz.2) & 201 & - & - & - & - & - \\
Ribas do Rio Pardo & 605 & 44,9 & 42,3 & 0,3 & 12,0 & 0,6 \\
São Gabriel do Oeste & 283 & 92,1 & 7,9 & 0 & 0 & 0 \\
Sidrolândia & 2.561 & 93,9 & 0,3 & 0 & 5,9 & 0 \\
Terenos & 2.074 & 95,3 & 4,8 & 0 & 0 & 0 \\
Média Geral & 1.731 & 80,3 & 9,3 & 4,7 & 3,1 & 2,6 \\
Amplitude das médias & $201-5.893$ & $35,3-100$ & $0-42,3$ & $0-49,8$ & $0-12,0$ & $0-14,3$
\end{tabular}

* Rebanho não submetido ao teste.

\section{Número de ovos por grama de fezes (OPG)}

A média de OPG antes do tratamento entre todos os rebanhos foi de 1.731. A maior contagem média foi constatada na propriedade localizada no município de Porto Murtinho (Faz.1) com média de 5.893 OPG, da qual foram recuperadas apenas larvas de Haemonchus spp. No mesmo município, na segunda propriedade visitada (Faz. 2), foi constatada a menor média, 201 OPG. Em virtude disso, o teste de redução não foi realizado nesse rebanho.

\footnotetext{
${ }^{7}$ Valbazen $^{\circledR}$, Laboratórios Pfizer Ltda, Rua Alexandre Dumas 1711, Chácara Santo Antonio, São Paulo, SP.

8 Trimix ${ }^{\text {TM }}$, Merial Saúde Animal Ltda, Av. Carlos Grimaldi 1701, São Quirino, Campinas, SP.

${ }^{9}$ Galgosantel $^{\circledR}$, Biogénesis Bago Saúde Animal Ltda, Rua Del. Leopoldo Belczak 286, Capão da Imbuia, Curitiba, PR.

${ }^{10}$ Ivomec $^{\circledR}$, Merial Saúde Animal Ltda, Av. Carlos Grimaldi 1701, São Quirino, Campinas, SP.

${ }^{11}$ Ripercol ${ }^{\circledR}$, Fort Dodge Saúde Animal Ltda, Rua Luiz Fernando Rodrigues 1701, Vila Boa Vista, Campinas, SP.

${ }^{12}$ Cydectin $^{\circledR}$, Fort Dodge Saúde Animal Ltda, Rua Luiz Fernando Rodrigues 1701, Vila Boa Vista, Campinas, SP.

${ }^{13}$ Neguvon $^{\circledR}$, Bayer S.A., Rua Domingos Jorge 1100, Socorro, São Paulo, SP.
} 
Quadro 2. Percentagem de redução no número de ovos do tipo Strongylida por grama de fezes (OPG) após tratamentos com formulações anti-helmínticas comerciais (ALB: Albendazole; ALB+IVE+LEV: Albendazole, Ivermectina e Levamisole; CLO: Closantel; IVE: Ivermectina; LEV: Levamisole; MOX: Moxidectina; TRI: Triclorfon), nos rebanhos testados. Resultados analisados empregando o programa RESO*

\begin{tabular}{lccccccc}
\hline \multicolumn{1}{c}{ Rebanhos } & \multicolumn{7}{c}{ Moléculas } \\
\cline { 2 - 7 } & ALB & ALB+IVE+LEV & CLO & IVE & LEV & MOX & TRI \\
\hline Angélica & $-297,3$ & 52,0 & 3,0 & $-281,3$ & 23,5 & $-115,9$ & $-71,4$ \\
Camapuã (Faz. 01) & 68,7 & 100 & 87,3 & 8,5 & 98,2 & 1,4 & 21,1 \\
Camapuã (Faz. 02) & 66,5 & 24,5 & 84,0 & 30,2 & 54,7 & 71,7 & 89,6 \\
Campo Grande (Faz. 01) & 50,5 & 66,8 & 1,5 & 5,6 & 25,8 & 61,6 & 53,4 \\
Campo Grande (Faz. 02) & $-11,5$ & - & 60,8 & 44,6 & $-114,6$ & $-13,8$ & 50,8 \\
Corumbá & 16,3 & - & 71,5 & $-15,4$ & 86,8 & 96,5 & 90,2 \\
Coxim & 74,3 & 91,0 & 90,0 & 70,7 & $-54,4$ & 13,8 & 98,8 \\
Ivinhema & 71,2 & 64,6 & $-63,0$ & $-180,9$ & 43,1 & 57,3 & 72,7 \\
Miranda (Faz. 01) & 63,1 & - & 93,8 & 44,7 & 100 & 100 & 83,8 \\
Miranda (Faz. 02) & 36,8 & 97,7 & 97,4 & 62,1 & 88,5 & 0 & 88,5 \\
Porto Murtinho & 58,0 & 68,9 & 82,6 & 15,3 & 51,3 & 25,9 & 79,5 \\
Ribas do Rio Pardo & $-188,6$ & 100 & 24,1 & 60,9 & 60,4 & 20,8 & 42,5 \\
São Gabriel do Oeste & $-75,0$ & 57,1 & 56,3 & $-171,4$ & $-56,3$ & 0 & 87,5 \\
Sidrolândia & 77,1 & $-5,1$ & 82,5 & 18,0 & 52,7 & 42,2 & 99,3 \\
Terenos & 0 & $-47,4$ & $-24,5$ & $-5,3$ & $-29,3$ & 41,3 & 90,1 \\
Redução média & 0,7 & 55,8 & 49,8 & $-19,6$ & 28,7 & 26,8 & 65,1 \\
Rebanhos considerados sensíveis & $0 / 15(0 \%)$ & $3 / 12(25 \%)$ & $1 / 15(6,7 \%)$ & $0 / 15(0 \%)$ & $2 / 15(13,3 \%)$ & $2 / 15(13,3 \%)$ & $2 / 15(13,3 \%)$
\end{tabular}

* RESO -Analysis Program Version 2.01. CSIRO, Division of Animal Health, Glebe, NSW, Austrália.

\section{Redução na contagem de ovos após tratamentos}

Segundo os critérios propostos pela WAAVP (Coles et al. 1992); em todos os rebanhos testados, as formulações contendo Albendazol e Ivermectina apresentaram resultados considerados como resistentes, com médias de redução de 0,7 e -19,6\% respectivamente (Quadro 2). Closantel foi eficiente apenas no rebanho identificado como Miranda (Faz.2) com 97,4\%; nos demais não apresentou eficácia, com média geral de redução na contagem de OPG de 49,8\%.

As formulações contendo Levamisole, Moxidectina e Triclorfon, apresentaram eficácia em apenas dois rebanhos, com médias de redução de 28,$7 ; 26,8$ e $65,1 \%$, respectivamente. A associação de moléculas contendo Albendazol, Ivermectina e Levamisole foi eficaz na redução de OPG em três rebanhos de um total de 12 testados, com redução média de 55,8\%. Essa associação apresentou a maior média de eficácia das formulações, com $25 \%$ dos rebanhos testados apresentando populações sensíveis (Quadro 2).

Em oito rebanhos: Angélica, Camapuã (Faz.2), Campo Grande (Faz.1), Campo Grande (Faz.2), Ivinhema, Porto Murtinho, São Gabriel do Oeste e Terenos, totalizando $53,3 \%$ dos rebanhos, foi constada resistência múltipla, a todas as formulações testadas. Nos rebanhos onde houve eficácia, esta foi limitada a uma formulação (Corumbá, Coxim, Ribas do Rio Pardo e Sidrolândia), totalizando $26,67 \%$ dos rebanhos; ou a no máximo duas moléculas, correspondendo a 20\% dos rebanhos (Camapuã, Faz.1; Miranda, Faz.1 e Faz.2) (Quadro 2).
Quadro 3. Gêneros de nematóides identificados nas coproculturas pré e pós-tratamentos e os respectivos percentuais encontrados, em rebanhos ovinos no estado de Mato Grosso do Sul, testados para resistência a anti-helimínticos comerciais

\begin{tabular}{lccc}
\hline Gêneros & Pré-tratamento & Pós-tratamento & Diferença \\
\hline Cooperia sp. & 2,58 & 1,38 & $-46,51 \%$ \\
Haemonchus sp. & 80,28 & 80,33 & $+0,06 \%$ \\
Oesophagostomum sp. & 3,06 & 1,87 & $-38,89 \%$ \\
Strongyloides sp. & 4,74 & 2,42 & $-48,95 \%$ \\
Trichostrongylus sp. & 9,34 & 14,01 & $+50,00 \%$
\end{tabular}

Gêneros de helmintos resistentes identificados nas coproculturas pós-tratamentos

Dois gêneros foram identificados como predominantemente resistentes nos rebanhos: Haemonchus sp. e Trichostrongylus sp. (Quadro 3). Os demais reduziram sua presença de forma significativa; porém a sobrevivência de alguns indivíduos é indicativa de que a frequência gênica para a resistência tem potencial para aumentar nas amostras populacionais em estudo.

Haemonchus sp. foi o gênero que apresentou a maior percentagem de resistência, com média de $86,9 \%$; a segunda maior percentagem foi de Trichostrongylus sp., com média de 47,5\%, Strongyloides sp., Oesophagostomum $\mathrm{sp}$. e Cooperia sp. também apresentaram amostras resistentes, com média de 33,6; 21,4 e 19,7\%, respectivamente (Quadro 4). 
Quadro 4. Percentuais da condição de resistência (R), baixa resistência (BR) e sensibilidade (S) dos gêneros de nematóides, à diferentes princípios ativos, encontrados em rebanhos ovinos no Estado de Mato Grosso do Sul; identificados pelo teste de redução na contagem de ovos após tratamentos, calculados pelo programa RESO* e coproculturas

\begin{tabular}{|c|c|c|c|c|c|c|}
\hline \multirow[t]{2}{*}{ Moléculas } & \multicolumn{6}{|c|}{ Gêneros } \\
\hline & & Cooperia sp. & Haemonchus sp. & Trichostrongylus sp. & Oesophagostomum sp. & Strongyloides sp. \\
\hline \multirow[t]{3}{*}{ Albendazole } & $\mathrm{R}$ & 16,7 & 100 & 58,3 & 25,0 & 20,0 \\
\hline & $\mathrm{BR}$ & 33,3 & 0 & 33,3 & 25,0 & 40,0 \\
\hline & $S$ & 50,0 & 0 & 8,3 & 50,0 & 40,0 \\
\hline \multirow[t]{3}{*}{ Alb+lver+Lev } & $\mathrm{R}$ & 0 & 75,0 & 33,3 & 0 & 0 \\
\hline & $\mathrm{BR}$ & 50,0 & 8,3 & 11,1 & 0 & 0 \\
\hline & $S$ & 50,0 & 16,7 & 55,6 & 100 & 100 \\
\hline \multirow[t]{3}{*}{ Closantel } & $\mathrm{R}$ & 50,0 & 80,0 & 58,3 & 37,5 & 25,0 \\
\hline & $\mathrm{BR}$ & 33,3 & 0 & 25,0 & 50,0 & 50,0 \\
\hline & $S$ & 16,7 & 20,0 & 16,7 & 12,5 & 25,0 \\
\hline \multirow[t]{3}{*}{ Ivermectina } & $\mathrm{R}$ & 16,7 & 100 & 30,8 & 12,5 & 50,0 \\
\hline & $\mathrm{BR}$ & 33,3 & 0 & 23,1 & 12,5 & 0 \\
\hline & $S$ & 50,0 & 0 & 46,2 & 75,0 & 50,0 \\
\hline \multirow[t]{3}{*}{ Levamisole } & $\mathrm{R}$ & 14,3 & 86,7 & 69,2 & 75,0 & 40,0 \\
\hline & $\mathrm{BR}$ & 42,9 & 6,7 & 15,4 & 12,5 & 40,0 \\
\hline & $S$ & 42,9 & 6,7 & 15,4 & 12,5 & 20,0 \\
\hline \multirow[t]{3}{*}{ Moxidectina } & $\mathrm{R}$ & 0 & 86,7 & 36,4 & 0 & 50,0 \\
\hline & $\mathrm{BR}$ & 33,3 & 6,7 & 9,1 & 25,0 & 0 \\
\hline & $S$ & 66,7 & 6,7 & 54,5 & 75,0 & 50,0 \\
\hline \multirow[t]{3}{*}{ Triclorfon } & $\mathrm{R}$ & 40,0 & 80,0 & 46,2 & 0 & 50,0 \\
\hline & $\mathrm{BR}$ & 40,0 & 6,7 & 38,5 & 37,5 & 25,0 \\
\hline & $S$ & 20,0 & 13,3 & 15,4 & 62,5 & 25,0 \\
\hline \multirow[t]{3}{*}{ Média } & $\mathrm{R}$ & 19,7 & 86,9 & 47,5 & 21,4 & 33,6 \\
\hline & $\mathrm{BR}$ & 38,0 & 4,0 & 22,2 & 23,2 & 22,1 \\
\hline & $S$ & 42,3 & 9,0 & 30,3 & 55,4 & 44,3 \\
\hline
\end{tabular}

* RESO, Analysis Program Version 2.01. CSIRO, Division of Animal Health, Glebe, NSW, Austrália.

\section{DISCUSSÃo}

A predominância do parasitismo por Haemonchus contortus e Trichostrongylus colubriformis, relatada nesse estudo, foi semelhante às encontradas em necropsias por Kawano et al. (2001), Ramos et al. (1985) e Ramos et al. (2004), onde $H$. contortus também foi a espécie de maior frequência. Em relação às espécies de Cooperia spp., $C$. curticei apresentou a maior frequência, similar ao relatado por Buzzulini et al. (2007).

A prevalência de Haemonchus sp. e Trichostrongylus sp., nas coproculturas, foi semelhante, ao relatado por Vieira et al. (2008) e Pereira et al. (2008), em municípios do Rio Grande do Sul; assim como na revisão realizada por Farias et al. (1997), considerando estudos realizados na região Sul do Brasil. Chandrawathani et al. (1999), Nieto et al. (2003) e Fernandes et al. (2004) também relataram prevalência desses gêneros, sendo Haemonchus sp. o de maior representatividade.

A maior prevalência de Strongyloides sp., relatada para o rebanho identificado como Angélica (Quadro 1), possivelmente ocorreu devido a presença de animais jovens nos grupos experimentais. Entretanto, Ahid et al. (2008), no Rio Grande do Norte, identificaram $52,3 \%$ das larvas infectantes como pertencentes ao mesmo gênero, e relataram não ter encontrado diferenças quanto a percentagem de Strongyloides sp. nas coproculturas, comparando animais jovens e adultos.

A contagem média de 1.731 OPG, relatada nesse tra- balho; com amplitude das médias, nos rebanhos, variando de 201 a 5.893 OPG (Quadro 1), é superior aos resultados descritos por Melo et al. (2003), em estudo realizado no estado do Ceará e por Köse et al. (2007), na Turquia, que descreveram médias de 469 e de 868 OPG, respectivamente; e inferior aos resultados de Mwamachi et al. (1995), no Kênia e Soccol et al. (1996), no estado do Paraná, Brasil, com médias de 3.197 e 2.237, respectivamente. No rebanho identificado como Porto Murtinho (Faz.2), o teste de redução não foi realizado, em virtude da baixa contagem de OPG; esta situação foi atribuída ao tipo de manejo empregado, que consistia no pastejo consorciado com bovinos. Esse fato fortalece a proposta de Fernandes et al. (2004), que o pastejo rotacionado e consorciado com bovinos é eficaz na redução da infecção parasitária em ovinos.

A resistência dos helmintos em relação ao Albendazol também foi registrada por Gill (1996), Soccol et al. (1996) e Molento (2004). Com relação à molécula Ivermectina, não diferem do descrito por Soccol et al. (1996), Molento (2004) e Rosalinski-Moraes et al. (2007). Outros estudos revelam índices de resistência mais baixos (Melo et al. 2003, Álvarez-Sánchez et al. 2006, Traversa et al. 2007, Köse et al. 2007); mas considerando a situação desse fenômeno no mundo, é possível dizer que existe tendência de aumento nos níveis de resistência pela persistência do uso desta base, em virtude da cultura dos criadores em atribuir a ela alta eficiência facilitada pela redução do preço dessa formulação. 
A formulação contendo Closantel apresentou alto índice de resistência anti-helmíntica nos rebanhos testados, semelhante ao descrito por Soccol et al. (1996) e Chandrawathani et al. (1999). Mas oposto ao relatado por Echevarria et al. (1996), onde cerca de $80,5 \%$ das propriedades testadas foram consideradas portadoras de isolados de helmintos sensíveis a essa molécula.

A formulação contendo Levamisole foi eficiente na redução do OPG em dois rebanhos, indicando a presença de isolados de helmintos resistentes em $86,7 \%$ das propriedades testadas (Quadro 2), semelhante ao descrito por Echevarria et al. (1996); mas superiores aos encontrados por Melo et al. (2003), Rosalinski-Moraes et al. (2007) e Soccol et al. (1996), onde $41,2 \% ; 44,4 \%$ e $33,3 \%$ das propriedades estudadas apresentaram isolados de helmintos resistentes, respectivamente. E inferiores aos relatados por Gill (1996), Farias et al. (1997) e Traversa et al. (2007), onde a resistência foi relatada, respectivamente, em 100\%; 95,7\% e $100 \%$ dos rebanhos.

De forma semelhante apenas $13,3 \%$ dos rebanhos apresentaram isolados sensíveis à Moxidectina. RosalinskiMoraes et al. (2007), encontraram isolados sensíveis em apenas três das nove propriedades, testadas. Buzzulini et al. (2007), também testaram essa molécula, na forma injetável e não obtiveram reduções satisfatórias na contagem de OPG. Chandrawathani et al. (1999) relataram apenas suspeita de resistência anti-helmíntica à Moxidectina, em 38\% dos rebanhos estudados. Resultados distintos do relatado por Traversa et al. (2007), em que observaram redução de $100 \%$ da contagem de OPG em todos os rebanhos onde a molécula foi utilizada.

Apesar dos produtores terem relatado pouca utilização de formulações contendo Triclorfon, o número de propriedades que apresentaram populações de helmintos resistentes a essa molécula, foi de $86,7 \%$. Apenas dois rebanhos, Coxim e Sidrolândia apresentaram reduções de OPG com nível de eficiência de 98,8\% e 99,3\% (Quadro 2). Pereira et al. (2008), também identificaram resistência a essa molécula no estado do Rio Grande do Sul, onde a redução da OPG foi de $73 \%$. Esses resultados são, possivelmente, reflexos do uso desse fármaco no passado. Entretanto, foi a molécula que apresentou a maior redução média de OPG: $65,1 \%$, considerando todos rebanhos testados.

A associação composta por Albendazol, Levamisole e Ivermectina foi eficaz em $25 \%$ dos rebanhos testados (Camapuã, Faz.1; Miranda, Faz.2; e Ribas do Rio Pardo), com média geral de redução de OPG igual a 55,8\% (Quadro 2). Buzzulini et al. (2007), em estudo comparativo com a Moxidectina, utilizou uma formulação contendo essas mesmas moléculas e registrou redução na contagem de OPG de $99 \%$.

No rebanho Camapuã (Faz.1), a redução da OPG possivelmente foi causada por apenas uma das bases da combinação, Levamisole, visto que a mesma foi eficiente na formulação simples. Nos rebanhos identificados como Miranda (Faz.2) e Ribas do Rio Pardo, a associação das bases reduziu a contagem de OPG satisfatoriamente. Aparentemente, essa redução foi causada pela ação sinérgica entre as moléculas; visto que isoladamente não houve redução (Quadro 2). Entretanto, nos rebanhos Camapuã (Faz.2) e Sidrolândia, os resultados divergem, pois as bases em formulações simples, obtiveram reduções maiores do que aquela da associação. Nos rebanhos Angélica e São Gabriel do Oeste, o resultado foi inverso: a associação não reduziu a contagem de OPG para ser considerada eficaz, mas a redução foi maior do que aqueles das bases em formulações simples. Entrocasso et al. (2008) também relataram diferenças de redução de OPG entre a utilização de bases, seja separadamente ou em associação. Frente à diferença dos resultados e do contido na literatura, há necessidade de esclarecer se há ou não sinergismo das moléculas ou se estes foram resultados casuais.

Quanto aos gêneros identificados nas coproculturas após os tratamentos (Quadro 4), Ramos et al. (2002), em Santa Catarina, também identificaram altos índices de resistência em Haemonchus sp., em relação à Albendazol e Ivermectina, $74 \%$ e $100 \%$, respectivamente; entretanto, em relação a Closantel e Levamisole, a percentagem foi inferior a $20 \%$. Em relação ao Trichostrongylus sp. os mesmos autores identificaram $100 \%$ de sensibilidade do gênero à Ivermectina e Closantel e de 13 e $44 \%$ para a resistência à Albendazol e Levamisole, respectivamente. Rosalinski-Moraes et al. (2007) no mesmo estado, identificaram $100 \%$ de cepas resistentes de Haemonchus sp. à Ivermectina e à Moxidectina; porém sensíveis à Albendazol e Levamisole; Trichostrongylus sp., foi sensível à Ivermectina e à Moxidectina e resistente à Albendazol e Levamisole. No Ceará, Melo et al. (2003) também identificaram Haemonchus sp. e Trichostrongylus $\mathrm{sp}$. como os gêneros que apresentaram os maiores índices de resistência aos anti-helmínticos.

Resultados diferentes dos relatados por Echevarria et al. (1996), que testaram Closantel, Ivermectina e Levamisole em rebanhos ovinos no Rio Grande do Sul, e identificaram $20,7 \%$ e $19 \%$, respectivamente, de isolados de Haemonchus sp. resistentes a essas bases. No mesmo estudo, o gênero Trichostrongylus sp. foi sensível ao Closantel e apenas $2 \%$ dos rebanhos apresentaram resistência à Ivermectina e 5\% à Levamisole.

Os gêneros Strongyloides sp., Oesophagostomum sp. e Cooperia sp. também apresentaram amostras resistentes, com média de $33,6 \% ; 21,4 \%$ e $19,7 \%$, respectivamente (Quadro 4). Apesar de menor percentagem de amostras resistentes, esses dados sugerem que existe tendência de aumento na frequência gênica para a resistência anti-helmíntica nas populações examinadas.

Esses resultados demonstram que, Haemonchus contortus é a espécie de maior prevalência na região e a que apresentou maior índice de resistência a todas as moléculas anti-helmínticas testadas; o que pode acentuar o prejuízo aos ovinocultores, em virtude da sua característica de patogenia. 


\section{CONCLUSÕES}

As espécies de helmintos parasitas gastrintestinais de ovinos em Mato Grosso do Sul são: Cooperia curticei, Cooperia pectinata, Cooperia punctata, Haemonchus contortus, Oesophagostomum columbianum, Strongyloides papillosus e Trichostrongylus colubriformis. Sendo Haemonchus contortus, a mais prevalente.

Os resultados das contagens de OPG indicam que em Mato Grosso do Sul, o índice de contaminação dos rebanhos por parasitas gastrintestinais é alto, o que implica em prejuízos econômicos aos produtores, tanto pela mortalidade dos animais e/ou redução dos índices de produção de leite, carne e lã, quanto pelo custo dos frequentes tratamentos.

A resistência múltipla aos anti-helmínticos já está instalada na maioria dos rebanhos de ovinos do Estado de Mato Grosso do Sul. As espécies Haemonchus contortus e Trichostrongylus colubriformis foram as mais prevalentes nas coproculturas antes e depois dos tratamentos, indicando que suas populações possuem elevados índices de resistência às moléculas testadas.

Agradecimentos.- Ao CNPq e à Fundect pelo apoio financeiro; à Embrapa Gado de Corte pelo apoio logístico; aos laboratoristas Marco Antonio da Silva e Ananias Loveira pelo auxílio na execução deste trabalho; aos proprietários das fazendas onde os testes foram realizados e ao Frigorífico JS, pelo fornecimento dos animais de necropsia.

\section{REFERÊNCIAS}

Ahid S.M.M., Suassuna A.C.D., Maia, M.B., Costa V.M.M. \& Soares H.S. 2008. Parasitos gastrintestinais em caprinos e ovinos da região Oeste do Rio Grande do Norte, Brasil. Ciênc. Anim. Bras. 9:212218

Álvarez-Sánchez M.A., Pérez-Garcia J., Cruz-Rojo M.A. \& Rojo-Vásquez F.A. 2006. Anthelmintic resistance in trichostrongylid nematodes of sheep farms in Northwest Spain. Parasitol. Res. 99:78-83.

Amarante A.F.T., Barbosa M.A., Oliveira M.R., Carmello M.J. \& Padovani C.R. 1992. Efeito da administração de Oxfendazol, Ivermectina e Levamisole sobre os exames coproparasitológicos de ovinos. Braz. J. Vet. Res. Anim. Sci. 38(29):31-38.

Barreto M.A., Almeida M.A.O., Silva A., Rebouças I. \& Mendonça L. R. 2002. Eficácia anti-helmíntica do cloridrato de Levamisole, Albendazol e Ivermectina em caprinos, na região semi-árida da Bahia. Anais $12^{\circ}$ Seminário Brasileiro de Parasitologia Veterinária, Rio de Janeiro, RJ. (Resumo)

Barreto M.A. \& Silva J.S. 1999. Avaliação da resistência de nematódeos gastrintestinais em rebanhos caprinos do Estado da Bahia: resultados preliminares. Anais $9^{\circ}$ Seminário Brasileiro de Parasitologia Veterinária, Salvador, BA. 160p.

Bispo M.J., Faustino M.A.G., Lima M.M., Oliveira G.A., Ramos C.A.N. \& Bispo C.A.S. 2002. Avaliação do tratamento anti-helmíntico com Oxfendazole e Ivermectina em rebanho caprino do Instituto Xingó, Piranhas, AL. Anais do $12^{\circ}$ Seminário Brasileiro de Parasitologia Veterinária, Rio de Janeiro, RJ.

Brasil 2007. Produção da Pecuária Municipal (PPM). Vol.35. Instituto Brasileiro de Geografia e Estatística (IBGE), Ministério do Planejamento, Orçamento e Gestão, Brasília, DF.

Buzzulini C., Silva-Sobrinho A.G., Costa A.J., Santos T.R., Borges F.A. \& Soares V.E. 2007. Eficácia anti-helmíntica comparativa da associação Albendazol, Levamisole e Ivermectina à Moxidectina em ovinos. Pesq. Agropec. Bras. 42(6):891-895.
Chandrawathani P., Adnan M. \& Waller P.J. 1999. Anthelmintic resistance in sheep and goat farms on Peninsular Malaysia. Vet. Parasitol. 82:305-310.

Coles G.C., Bauer C., Borgsteede F.H.M., Geerts S., Klei T.R., Taylor M.A. \& Waller P.J. 1992. World Association for the Advancement of Veterinary Parasitology (WAAVP) methods for detection of anthelmintc resistance in nematodes of veterinary importance. Vet. Parasitol. 44:3544

Cunha-Filho L.F.C., Pereira A.B.L. \& Yamamura M.H. 1998. Resistência a anti-helmínticos em ovinos na região de Londrina, PR, Brasil. Semina 19(1):31-37.

Echevarria F.A.M. 1988. Doenças parasitárias de ovinos e seu controle. Anais do 3ํㅗำósio Paranaense de Ovinocultura, Londrina, PR, p.46-47.

Echevarria F., Borba M.F.S., Pinheiro A.C., Waller P.J. \& Hansen J.W. 1996. The prevalence of anthelmintic resistance in nematode parasites of sheep in Southern Latin America: Brazil. Vet. Parasitol. 62:199206.

Entrocasso C., Alvarez L., Manazza J., Lifschitz A., Borda B., Virkel G., Mottier L. \& Lanusse C. 2008. Clinical efficacy assessment of the Albendazol-Ivermectin combination in lambs parasitized with resistant nematodes. Vet. Parasitol. 155:249-256.

Farias M.T., Bordin E.L., Forbes A.B. \& Newcomb K. 1997. A survey on resistance to anthelmintic in sheep stud farms of southern Brazil. Vet. Parasitol. 72:209-214

Fernandes L.H., Seno M.C.Z., Amarante A.F.T., Souza H. \& Belluzzo C.E.C. 2004. Efeito do pastejo rotacionado e alternado com bovinos adultos no controle da verminose em ovelhas. Arq. Bras. Med. Vet. Zoo. 56(6):733-740.

FAO 2003. Resistencia a los antiparasitarios: estado actual con énfasis en América Latina. Food and Agriculture Organization of the United Nations, Rome. 51p.

Gennari S.M., Vieira Bressan M.C.R., Rogero J.R., MacLean J.M. \& Duncan J.L. 1991. Pathophysiology of Haemonchus placei infection in calves. Vet. Parasitol. 38:163-172.

Gill B.S. Anthelmintic resistance in India. 1996. Vet. Parasitol. 63:173176

Gordon H.M.L. \& Whitlock H.N. 1939. A new technique for counting nematode egg in sheep faeces. J. Commonw. Sci. Ind. Organ. 12(1):50-52.

Hammerberg D.V.M. 1986. Pathophysiology of Nematodiasis in Cattle. Vet. Clin. North Am., Food Anim. Pract. 2(2):225-234.

Holmes P.H. 1987. Pathophysiology of parasitic infections. Parasitology 94:29-51.

Jardim W.R. 1974. Os Ovinos. Nobel, São Paulo. 196p.

Kawano E.L., Yamamura M.H. \& Ribeiro E.L.A. 2001. Efeitos do tratamento com anti-helmíntico em cordeiros naturalmente infectados com helmintos gastrintestinais sobre os parâmetros hematológicos, ganho de peso e qualidade da carcaça. Arq. Fac. Vet. UFRGS 29(2):113121.

Keith R.K. 1953. The differentiation of the infective larvae of some commum nematode parasite of cattle. Aust. J. Zool. 1(2):223-235.

Köse M., Kozan E., Sevimli F.K. \& Eser M. 2007. The resistance of nematode parasites in sheep against anthelmintic drugs widely used in Western Turkey. Parasitol. Res. 101:563-567.

Lichtenfels J.R. \& Pritchard M.H. 1982. A guide to the parasite collections of the world. Lawrence, American Society of Parasitologists, Lincon, NE. 79p.

Melo A.C., Reis I.F., Bevilaqua C.M.L., Vieira L.S., Echevarria F.A.M. \& Melo L.M. 2003. Nematódeos resistentes a anti-helmínticos em rebanhos de ovinos e caprinos do estado do Ceará, Brasil. Ciência Rural 33:339-344.

Melo A.C.F.L., Bevilaqua C.M.L., Villaroel A.S. \& Girão M.D. 1998. Resistência a anti-helmínticos em nematóides gastrintestinais de ovinos 
e caprinos, no município de Pentecoste, Estado do Ceará. Ciênc. Anim. 8:7-11.

Molento M.B. 2004. Resistência de helmintos em ovinos e caprinos. Revta Bras. Parasitol. Vet. 13(1):82-87.

Mwamachi D.M., Audho J.O., Thorpe W. \& Baker R.L. 1995. Evidence for multiple anthelmintic resistance in sheep goats reared under the same management in coastal Kenya. Vet. Parasitol. 60:303-313.

Nieto L.M., Martins E.N., Macedo F.A.F. \& Macedo Zundt M. 2003. Observações epidemiológicas de helmintos gastrintestinais em oveIhas mestiças manejadas em pastagens com diferentes hábitos de crescimento. Ciênc. Anim. Bras. 4(1):45-51.

Pereira R.C.F., Toscan G., Vogel F.S.F. \& Sangioni L.A. 2008. Resistência de helmintos gastrointestinais em ovinos de Rosário do Sul, RS, Brasil. Anais $35^{\circ}$ Congresso Brasileiro de Medicina Veterinária, Gramado, RS. CD-ROM (Resumo)

Presidente P.J.A. 1985. Methods for detection of resistance to anthelmintics, p.13-28. In: Waller P.J. \& Anderson N. (Eds), Resistance in Nematodes to Anthelmintic Drugs. CSIRO, Division of Animal Health, Glebe, NSW, Australia.

Ramos C.I., Bellato V., Ávila V.S., Coutinho G.C. \& Souza A.P. 2002. Resistência de parasitos gastrintestinais de ovinos a alguns anti-helmínticos no Estado de Santa Catarina, Brasil. Ciência Rural 32:473477.

Ramos C.I., Bellato V., Souza A.P., Ávila V.S., Coutinho G.C. \& Dalagnoll C.A. 2004. Epidemiologia das helmintoses gastrintestinais de ovinos no Planalto Catarinense. Ciência Rural 34(6):1889-1895.

Ramos C.I., Paloschi C.G., Perussolo S. \& Freitas R. 1985. Gastrointestinal and pulmonary helminths in sheep on the Santa Catarina Plateau. Abstracts $2^{\text {nd }}$ Conference World Association for Advancement of Veterinary Parasitology (WAAVP), Rio de Janeiro, $\mathrm{RJ}, \mathrm{s} / \mathrm{p}$.

Roberts F.H.S. \& O'Sullivan J.P. 1950. Methods for egg counts and larval cultures for strongyles infesting the gastrointestinal tract of cattle. Aust. J. Agric. Res. 1:99-102.
Rosalinski-Moraes F., Moretto L.H., Bresolin W.S., Gabrielli I., Kafer L., Zanchet I.K., Sonaglio F. \& Thomaz-Soccol V. 2007. Resistência antihelmíntica em rebanhos ovinos da região da Associação dos Municípios do Alto Irani (AMAI), Oeste de Santa Catarina. Ciênc. Anim. Bras. 8(3):559-565.

Santiago M.A.M. \& Costa U.C. 1979. Resistência de Haemonchus contortus, Trichostrongylus colubriformis e Ostertagia spp, ao Levamisole. Revta Cent. Ciênc. Rurais, UFSM, 9:315-318.

Santos V.T. \& Gonçalves P.C. 1967/68. Verificação de estirpes de Haemonchus contortus resistentes ao Thiabendazole no Rio Grande do Sul, Brasil. Revta Fac. Agron. Vet. UFRGS 9:201-211.

Soccol V.T., Sotomaior C., Souza F.R. \& Castro E.A. 1996. Occurrence of resistance to anthelmintics in sheep in Paraná state, Brazil. Vet. Rec. 139:421-422.

Traversa D., Paoletti B., Otranto D. \& Miller J. 2007. First report of multiple drug resistance in trichostrongyles affecting sheep under field conditions in Italy. Parasitol. Res. 101:1713-1716.

Veríssimo C.J., Oliveira S.M. \& Filha E.S. 2002. Eficácia de alguns antihelmínticos em uma ovinocultura no Estado de São Paulo, Brasil. Anais $12^{\circ}$ Seminário Brasileiro de Parasitologia Veterinária, Rio de Janeiro, RJ, s/p.

Vieira L.S., Berne M.E.A. \& Cavalcante A.C.R. 1989. Redução do número de ovos por grama de fezes (OPG) em caprinos medicados com anti-helmínticos. Boletim de Pesquisa 11, Embrapa. 24p.

Vieira M.I.B., Rocha H.C., Ractz L.A.B., Nadal R., Moraes R.B. \& Oliveira I.S. 2008. Comparação de dois métodos de controle de nematódeos gastrintestinais em borregas e ovelhas de corte. Ciênc. Agrar. 29(4):853-860.

Wood I.B., Amaral N.K., Bairden K., Duncan J.L., Kassai Y., Malone J.B. Jr, Pankavich J.A., Reinecke R.K., Slocombe O., Taylor S.M. \& Vercruysse J. 1995. World Association for the Advancement of Veterinary Parasitology (WAAVP) Second edition of guidelines for evaluating the efficacy of anthelmintics in ruminants (bovine, ovine, caprine). Vet. Parasitol. 58:181-213. 